\title{
Fox Chase SCID Beige Mouse
}

National Cancer Institute

\section{Source}

National Cancer Institute. Fox Chase SCID Beige Mouse. NCI Thesaurus. Code C123644.

A CB17 SCID mouse with an additional mutation on the Lyst gene which results in defective natural killer cells. 